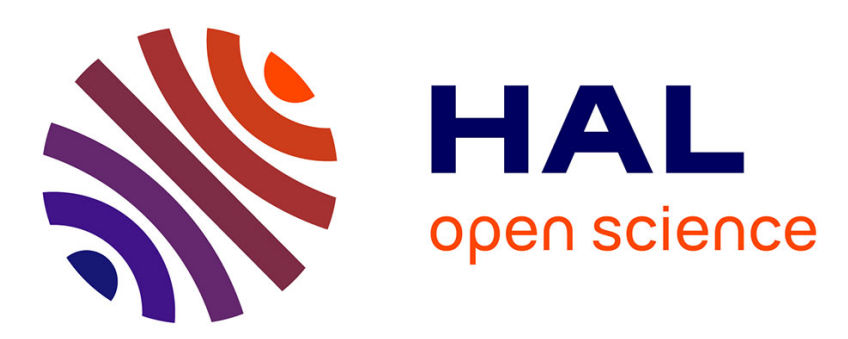

\title{
Comment expliquer les erreurs des multinationales et de leurs consultants?
}

\author{
Michel Villette
}

\section{To cite this version:}

Michel Villette. Comment expliquer les erreurs des multinationales et de leurs consultants?. Gestion - HEC Montréal, 2009, 34 (3), pp.13 - 17. halshs-01392753

\section{HAL Id: halshs-01392753 \\ https://shs.hal.science/halshs-01392753}

Submitted on 4 Nov 2016

HAL is a multi-disciplinary open access archive for the deposit and dissemination of scientific research documents, whether they are published or not. The documents may come from teaching and research institutions in France or abroad, or from public or private research centers.
L'archive ouverte pluridisciplinaire HAL, est destinée au dépôt et à la diffusion de documents scientifiques de niveau recherche, publiés ou non, émanant des établissements d'enseignement et de recherche français ou étrangers, des laboratoires publics ou privés. 


\title{
Article publié dans la Reuve Internationale de Gestion,
}

Automne 2009, Volume 34, N³, HEC Montréal, CANADA, p.13-17.

\section{Comment expliquer les erreurs des multinationales et de leurs consultants?}

\author{
Michel Villette, professeur de sociologie à AgroParisTech et chercheur associé au Centre Maurice \\ Halbwachs, ENS-EHESS-CNRS, Paris.
}

\section{Résumé}

Cette réflexion porte sur certains facteurs expliquant l'apparition d'erreurs dans la chaîne de transmission qui va des sièges mondiaux des grands groupes jusqu'aux établissements décentralisés implantés dans divers pays, en passant par les cabinets de conseil. Ces facteurs comprennent notamment la distance géographique et culturelle favorisant une solution trop rapide; une définition simpliste du bien commun; la quête de performances exceptionnelles par l'entremise de restructurations et de réformes constantes; le souci et l'importance de bien paraitre, d'obéir et de se conformer; les impératifs de rentabilité des sociétés de conseil et des autres intermédiaires. La réflexion traite de l'impact moral que cette situation a sur les jeunes diplômés qui débutent dans les métiers de conseil de même que sur les cadres intermédiaires et les employés. Il est possible de limiter le nombre des erreurs en insistant sur la nécessité de doser interventionnisme et contrôles pour favoriser la capacité d'innovation et d'adaptation des organisations. 
omment expliquer les crises économiques et financières? D'un point de vue sociologique, il importe de reconnaître qu'une série d'erreurs radicales et persistantes (Morel, 2002) sont commises le long de la chaîne de commandement, qui va des sièges mondiaux des grandes multinationales, des banques et des cabinets de conseil internationaux jusqu'aux établissements industriels et commerciaux de base, dispersés dans de nombreux pays. Sans prétendre à l'exhaustivité, on peut relever les facteurs principaux qui expliquent ces erreurs. Cette réflexion vise à préciser certaines de ces causes. Nous insistons ici sur cinq grandes causes sans établir de priorités, car chacune d'elles est importante.

\section{La distance géographique et culturelle favorisant une solution trop rapide}

Comme l'a montré Mintzberg (1989), on observe parmi les P.D.G. de société une tendance à appliquer des formules toutes faites, des techniques standardisées pour résoudre un problème qui concerne une de leurs nombreuses filiales, sans prendre en compte les particularités de la situation locale. Par manque de temps, ignorance ou indifférence, seuls quelques indicateurs chiffrés sont examinés. Le côté humain et les difficultés de la conduite effective des opérations sont négligés. Ainsi, pour prendre un exemple, un chercheur finlandais a montré que le temps consacré aux négociations budgétaires au sein d'une multinationale était d'autant plus court que la distance était grande entre le siège mondial et la filiale.

Cette propension à «normaliser» les activités au travail est renforcée par le type de formation qu'ont reçue les consultants, en particulier ceux qui sont passés par le MBA des grandes écoles de gestion (Mintzberg, 2004). Les jeunes gens issus de ces formations et leurs professeurs imposent une forme symbolique standardisée pour penser ce qu'est, et ce que doit être, une entreprise qui fonctionne «bien». Brunsson (2006) a présenté avec beaucoup d'humour les artifices qui permettent à un gestionnaire ou à un consultant d'éviter d'aborder les conséquences pratiques de ce qu'il décide dans l'urgence et sur la base 
d'informations abstraites. Typiquement, les experts en gestion modernes prennent leurs décisions sur la base de tableaux chiffrés, de discours et de théories. Ils s'informent sur la pratique à partir de ce que les praticiens en disent et non pas d'une observation minutieuse sur le terrain. Ces experts concentrent leur intérêt sur l'avenir, ce qui reporte toute confrontation avec la pratique au-delà de l'échéance à laquelle ils doivent rendre des comptes. Ils ne parlent jamais de leur propre pratique, mais de celle des autres, et lorsqu'une pratique s'accorde mal avec leurs théories, ils disent que c'est un cas particulier, ou alors ils changent de théorie et de méthode si fréquemment qu'il est impossible de les soumettre sérieusement à l'épreuve du réel.

Il importe aussi de traiter de la créativité en matière de présentation des comptes, créativité qui est source d'illusions dont peuvent être dupes, pendant une certaine période, les investisseurs boursiers. Sur ce point, on ne saurait trouver un meilleur exemple que la faillite du négociant en énergie Enron en 2002 et la disparition du cabinet d'audit international Andersen, qu'il a entraîné dans sa chute (Surowiecki, 2002: 190-196; 209-218). Facteur facilitant et aggravant le précédent, les systèmes d'information donnent aux dirigeants du siège mondial l'illusion qu'ils peuvent tout connaître, contrôler et manipuler à distance. Les tableaux chiffrés qui apparaissent sur les ordinateurs sont trop souvent considérés comme une image assez fiable, précise et complète de la réalité.

\section{Une définition simpliste du bien commun}

Un autre facteur expliquant l'apparition d'erreurs dans la chaîne de transmission est la tendance des dirigeants de compagnies internationales à s'en tenir à une définition simpliste du bien commun. Kimberly (1980) a indiqué les éléments constitutifs de cette définition simpliste. Quelles sont les expressions typiquement utilisées pour décrire une entreprise hautement performante? «En croissance rapide», «très rentable», «capable de s'adapter». Quels sont les attributs qu'on associe habituellement aux entreprises les plus performantes? «Productivité élevée», «de grande taille», «part du marché élevée», «personnel exceptionnel», «bonne gestion». Si l'on combine ces expressions et ces attributs, quel type 
d'organisation se trouve décrit? Kimberly (1980; traduction libre) apporte une réponse sans équivoque: «Une organisation qui contrôle son champ d'activité, qui contrôle sa technologie, qui contrôle sa structure, qui contrôle son échelle d'opérations, qui contrôle ses environnements interne et externe.» Ces formes de contrôle impliquent que l'organisation est relativement autonome, libre des contraintes que pourraient lui imposer d'autres organisations (par exemple, l'administration), libre d'obligations vis-à-vis des tiers, sans engagements à respecter. Aujourd'hui, on entend beaucoup de discours sur la responsabilité sociale des entreprises et sur la nécessité d'assurer un développement durable respectueux des parties prenantes et de l'environnement. Cependant, on peut douter que la définition de la performance organisationnelle ait vraiment changé. D'ailleurs, les dirigeants rappellent souvent que l'atteinte des objectifs économiques est une condition - préalable et non négociable - de la poursuite d'autres objectifs.

\section{La quête de performances exceptionnelles par l'entremise de restructurations et de réformes constantes}

Une étude publiée en 2007 dans The McKinsey Quarterly (Devan et al., 2007) indique qu'il est extrêmement rare (moins de $1 \%$ des cas) qu'une grande compagnie mondiale parvienne à maintenir sur une longue période (10 ans) à la fois une croissance et une rentabilité très supérieures à la moyenne des entreprises de même taille. De plus, ce résultat exceptionnel ( 9 cas seulement sur les 1077 compagnies de l'échantillon) s'expliquent par le pays et le type d'industrie plus que par la qualité exceptionnelle de la gestion. Néanmoins, beaucoup de dirigeants de grandes entreprises persistent à prévoir des résultats exceptionnels auxquels on ne peut parvenir qu'en prenant des risques excessifs, en cherchant à faire de l'argent facilement ou bien en imposant aux unités opérationnelles des objectifs irréalistes. De tels objectifs généreront d'ailleurs du stress chez les employés, une baisse de la qualité des services rendus aux clients et une perte de compétitivité de l'entreprise à moyen terme, parce que des aspects importants comme l'innovation ou l'attention aux clients auront été négligés au profit de résultats à court terme. 
Le souci de faire mieux que les concurrents et d'obtenir des résultats financiers exceptionnels pousse les dirigeants des grandes sociétés mondiales à lancer de perpétuelles réformes : réorganisations, restructurations et autres programmes d'amélioration de la productivité, de la qualité, etc. Le rythme de ces réformes est souvent beaucoup trop rapide pour qu'on ait le temps d'en vérifier le bien-fondé et de corriger leurs éventuels défauts de conception. Tout se passe comme si on lançait une nouvelle réforme avant que les inconvénients de la précédente ne deviennent trop apparents. On a parlé à ce propos de «mode de gestion» et les travaux de Midler (1986) et Abrahamson (1999) en ont montré le côté profondément irrationnel.

Une des caractéristiques principales des réformes, c'est qu'elles obligent les membres de l'entreprise à se conformer à un modèle officiel, prescrit par les dirigeants. Elles tendent à obliger chacun à rendre ce qu'il fait conforme à ce qui est dit que l'on doit faire. La norme, efficace ou non, applicable ou non, dangereuse ou non, doit être appliquée. Les réformes n'aident pas les personnes au travail à échapper à la règle ; au contraire, elle les oblige à prendre la règle en compte. Très souvent, ce sont des consultants ou des auditeurs qui viennent imposer la règle et contrôler son application. Leur recommandation peut se résumer en un slogan, d'ailleurs couramment utilisé : «Dites ce que vous faites, faites ce que vous dites.» Avant une intervention, le consultant conclut toujours que les choses vont mal : il faut donc une réforme! Après son intervention, il affirme que tout va mieux : il faudra donc intervenir de nouveau pour progresser encore! Si, par malchance, il lui faut admettre que l'intervention a échoué, il dira que le principe était bon et la méthode excellente, mais que les conditions ont été défavorables. Une nouvelle intervention s'avère donc nécessaire!

\section{$\underline{\text { Le souci et l'importance de bien paraître, d'obéir et de se conformer }}$}

Dans les grands groupes mondiaux, il arrive que le responsable local d'un établissement ou d'une filiale conteste l'analyse du siège mondial et s'oppose aux décisions venues d'en haut (pour un exemple, voir Goldratt et Cox, 1984). Dans ce cas, il est assez probable, mais pas fatal, qu'il sera muté ou remercié, 
et qu'on enverra des consultants ou des «gestionnaires de transition» exécuter coûte que coûte la décision de l'état-major central.

Pour résoudre un problème, tel que la direction l'a perçu à travers quelques indicateurs chiffrés, on fait appel à un cabinet de consultants, choisi parce qu'il prétend apporter une «solution» au problème de la direction (par exemple, le manque de productivité d'un atelier, une rentabilité insuffisante des capitaux investis, une qualité insuffisante de la production). À son tour, après un diagnostic rapide (parfois après seulement quelques heures d'entretiens avec les principaux responsables), le cabinet de conseil envoie en mission sur place de jeunes consultants pleins de bonne volonté, désireux de démontrer au plus vite ce dont ils sont capables et tout juste sortis d'une école de gestion. Ces jeunes gens, anxieux à l'idée de faire mauvaise figure, se rassurent en mettant en œuvre des solutions techniques préétablies, prescrites par le cabinet qui les emploie ou apprises à l'école (Berry, 1991). Le souci de se conformer aux prescriptions, plutôt que de chercher à comprendre les particularités de la situation rencontrée et à s'y adapter, va produire toutes sortes de catastrophes imperceptibles, dont la gravité n'apparaîtra souvent qu'après leur départ. En effet, ces jeunes gens ne vont pas rester bien longtemps dans l'entreprise, ils n'auront pas à subir les effets négatifs à long terme des solutions qu'ils prescrivent. Ils ne pourront pas non plus apprendre de leurs erreurs en se rendant compte de leurs conséquences cachées. Ils s'en tiendront à l'apparence trompeuse des résultats mesurables à court terme, dont ils pourront se targuer pour avancer dans leur carrière.

Je ne prétends pas que les conseillers en gestion font seulement un «sale boulot». Je soutiens cependant que la plupart d'entre eux font un sale boulot à un moment ou à un autre de leur carrière, comme j'en ai d'ailleurs fait l'expérience (Villette, 1988). À mon avis, la profession de consultant doit être étudiée avec attention d'un point de vue sociologique, parce qu'elle constitue un symptôme des dysfonctionnements du système économique mondial. Dans beaucoup de pays, dont la France (Villette, 1988, 2003; Moutout, 2003), le Royaume-Uni (O'Shea et Madigan, 1997; Power, 1997) et les États-Unis 
(Perkins, 2004; Surowiecki, 2002), des controverses ont éclaté autour du métier de conseil. Sans faire référence ici aux opérations auxquelles j'ai participé et qui visaient à rendre «acceptable» aux populations riveraines et aux élus locaux l'implantation de centrales nucléaires sur le territoire français (Villette, 1988), je citerai un cas présenté par O'Shea et Madigan. En Alabama, en 1994, le cabinet de conseil Andersen Consulting s'était engagé par contrat, auprès de l'entreprise métallurgique O'Neal, à réduire les effectifs salariés de 100 personnes au moins. Comme le cabinet de conseil n'est pas parvenu à atteindre ce chiffre, O’Neal a exigé qu'il paie la pénalité prévue au contrat. Devant le refus d'Andersen, O'Neal lui a intenté un procès. Ce procès est particulièrement éclairant en ce qu'il considère comme un comportement «déviant» non pas le fait pour un cabinet de conseil d'être payé pour exclure 100 salariés du marché du travail, mais le fait de ne pas être arrivé à obtenir ce résultat!

\section{Les impératifs de rentabilité des sociétés de conseil et des autres intermédiaires}

La rentabilité d'une société de conseil dépend essentiellement de l'utilisation à plein temps de sa force de travail. Il y a donc une pression permanente exercée sur chacun de ses membres pour qu'il facture un maximum de ses journées de travail à des clients. Il y a aussi une pression permanente exercée sur les associés et les consultants pour qu'ils négocient des contrats permettant d'utiliser au maximum la force de travail disponible. C'est ainsi que trop souvent de jeunes consultants sont affectés à des missions pour lesquelles ils n'ont aucune compétence spécifique, simplement parce qu'ils sont inemployés au moment où le contrat démarre. On leur impose alors d'accomplir «en experts» une tâche qu'ils effectuent pour la première fois (Villette, 1988).

On peut dire que dans l'industrie du conseil en gestion, les impératifs commerciaux déterminent la production de connaissances, et non pas l'inverse. Être consultant consiste principalement à pouvoir devenir presque instantanément «expert» dans les sujets pour lesquels il y a une demande. La diversité de cette demande rend très vite caduc le savoir que l'on a acquis dans une phase antérieure de sa carrière. Pour faire coïncider l'offre et la demande d'expertise, il faut donc user d'expédients et d'artifices 
rhétoriques. Être un «beau parleur» devient un trait de caractère typique du consultant vieillissant (Villette, 2003). Il y a un autre aspect du métier de consultant qu'il faut considérer: plus un consultant est payé cher, plus il est censé agir vite et avec aplomb, avant d'avoir eu le temps de bien comprendre la situation sur laquelle il doit intervenir. C'est ainsi qu'il est souvent conduit à formuler des prescriptions dans l'ignorance de leurs limites et conditions d'emploi.

Reconnaissons aussi que les consultants, les cabinets d'avocats d'affaires, les organismes de prévisions économiques et les banquiers ont globalement tendance à privilégier le point de vue de leurs meilleurs clients, les plus riches, les plus influents, au détriment des clients moins solvables et moins susceptibles d'apporter des affaires intéressantes. Ils ont aussi intérêt à pousser leurs gros clients à multiplier les opérations apportant des honoraires. Ainsi, en dépit des codes de déontologie et autres chartes d'éthique régulièrement affichées par ces professions, on pourrait formuler les propositions suivantes :

- Plus les changements de propriété des établissements industriels et commerciaux sont fréquents, plus les intermédiaires (juristes, consultants et banquiers) font de bonnes affaires.

- Plus la distance (géographique et culturelle) est grande entre les propriétaires et les entreprises, plus les intermédiaires trouvent d'occasions d'effectuer des missions.

- Plus il y a de dysfonctionnements provoqués par l'application standardisée des normes de la gestion internationale, plus ces normes changent vite et plus il est nécessaire de faire de fréquentes réformes pour que les entreprises puissent être évaluées, certifiées, auditées et, donc, faciles à revendre à de nouveaux propriétaires.

- Plus les intermédiaires sont influents, plus ils ont su se rendre indispensables et imposer partout leurs standards et leurs perpétuels changements de définition des standards, et plus cela facilite et encourage les changements fréquents de propriété.

\section{Implications et défis}


Les erreurs et leurs causes ne sont pas sans incidences. Une souffrance et une détresse coûteuses sont vécues par bien des acteurs. Comment limiter le nombre des erreurs ou promouvoir l'éthique dans la gouvernance des sociétés internationales? En insistant particulièrement sur la nécessité de doser avec tact interventionnisme et contrôles pour favoriser la capacité d'innovation et d'adaptation des organisations.

\section{Une souffrance et une détresse coûteuses}

Apprendre à devenir très vite un expert sur n'importe quel sujet, toujours paraître porteur des vérités de l'expertise présente un coût psychologique certain. Exécuter à la lettre la mission prévue au contrat, même lorsqu'elle semble défier le bon sens ou la morale commune, peut être une expérience traumatisante pour les consultants débutants. De même, toujours promettre des résultats positifs, quel que soit le projet et quels que soient les doutes susceptibles de naître une fois que l'on a appris à connaître la situation d'un peu plus près et que l'on découvre tout ce que les rédacteurs du contrat ont ignoré ou négligé est aussi une source de stress et de confusion (Villette, 1988). Pour les jeunes qui espèrent progresser rapidement dans leur carrière en tant que consultants, il est souvent difficile de prendre conscience avec lucidité de la part de mystification qu'implique leur statut d'expert (Berry, 1991). Pour réussir, beaucoup considèrent qu'il vaut mieux ne pas trop se poser de questions. Comme le disait justement Bourdieu (1982) : «Si ceux qui ont partie liée avec l'ordre établi, quel qu'il soit, n'aiment guère la sociologie, c'est qu'elle introduit une liberté par rapport à l'adhésion primaire qui fait que la conformité même prend un air d'hérésie ou d'ironie.»»

Une manière judicieuse pour les consultants d'éviter cet écueil serait de suivre les préceptes classiques du «processus de consultation» tels que les a énoncé Schein (1987). Suivant cette méthodologie en vogue dans les années 1980, le consultant pourrait «recadrer» la demande du client et le sensibiliser aux implications de ses choix. Il ne s'approprierait pas le problème de l'organisation cliente, mais il se définirait comme un «accompagnateur» spécialiste d'une méthodologie d'intervention laissant le client «propriétaire» du problème et, par conséquent, le seul et véritable responsable de l'éventuel «sale 
boulot». Bien qu'elle reste intellectuellement séduisante, cette conception du consultant a été quantitativement dépassée au cours des années 1990 par les offres commerciales de cabinets qui ont «industrialisé» le conseil, proposé des «solutions», adopté plutôt une attitude d'expert, et même accepté d'être rémunérés en fonction des résultats à court terme de leurs interventions (Davenport, 1995; BerrebiHoffman, 2002).

Finalement, plus il y a d'erreurs le long de cette chaîne de transmission vouée au contrôle et à la coordination du commerce mondial, plus il y a de souffrance et de détresse parmi les employés et les gestionnaires de l'entreprise, parmi ses clients et parmi les consultants eux-mêmes. Ce sont ces petits groupes d'êtres humains qui vont avoir à subir le plus directement les conséquences des erreurs de fonctionnement du grand système. De nombreux diagnostics sociologiques ont montré les conséquences insidieuses à long terme d'un recours trop fréquent aux consultants pour mettre en œuvre d'incessantes réformes au sein des entreprises. Parmi les conséquences les plus fréquentes et les mieux analysées, on peut citer :

- le haut niveau de stress parmi les cadres moyens ;

- la difficulté croissante des employés à prendre des initiatives, à expérimenter, à tirer des enseignements personnels de leur expérience au travail pour progresser ;

- le manque de ressources (en temps de travail disponible et en budget) pour mettre au point des initiatives afin de mieux s'adapter au contexte local.

\section{Les défis pour les dirigeants}

Dans une perspective plus philosophique, on pourrait montrer que la gestion, dans la mesure où elle est animée d'une visée de contrôle, peut comporter une tendance au totalitarisme. Cette tendance est souvent limitée, en pratique, par l'imperfection des systèmes de contrôle, imperfection qui maintient une certaine opacité et protège en partie les unités décentralisées du regard de l'autorité centrale. Mais les progrès continus de l'informatisation et des réseaux de communication ne cessent de fournir de nouveaux 
moyens aux partisans du contrôle total. Supposons, à titre d'exercice de pensée, qu'un système d'information soit équipé d'un logiciel de gestion intégré parfait et parfaitement renseigné. La direction serait alors en mesure de réaliser l'idéal d'une entreprise «parfaitement gérée», c'est-à-dire que les résultats seraient exactement conformes aux objectifs fixés par les dirigeants (efficacité parfaite). Les résultats seraient atteints en consommant toujours un minimum de ressources (efficience parfaite) et aucun moyen ne serait utilisé à autre chose qu'à la poursuite des objectifs fixés par la direction (pertinence parfaite de l'allocation des moyens). On peut imaginer que cette entreprise utopique serait un univers peu propice à l'épanouissement des humains, réduit quasiment au rang d'automates. Mais cet argument humaniste semble aujourd'hui avoir peu de poids pour les adeptes de la technologie.

Plus embarrassant pour les dirigeants est le fait que cette organisation parfaitement efficace et efficiente, sans «mou dans les rouages» (organizationnal slack), serait incapable d'innover, de s'autotransformer pour s'adapter au changement du contexte des affaires. Or, l'instabilité et l'imprévisibilité des marchés obligent à préférer aujourd'hui des entreprises souples et adaptables, capables de performances moyennes dans des circonstances variées, plutôt que des entreprises parfaitement adaptées pour accomplir avec une efficacité maximale une tâche rigidement définie (Nitin et Ranjay, 1997). L'enjeu est donc de relâcher les contrôles, les audits et les prescriptions venus de l'extérieur, pour développer des entreprises capables de s'autoréformer en permanence, de s'adapter aux contextes locaux (Bartlett et Ghoshal, 1998) et d'apprendre à apprendre.

\section{Références}

Abrahamson, E. (1999), «Management fashion: Life circles, triggers and collective learning processes», Administrative Sciences Quarterly, décembre.

Bartlett, C.A., Ghoshal, S. (1998), Managing across Borders: The Transnational Solution, Harvard Business School Press.

Berrebi-Hoffman, I. (2002), «Les multinationales du conseil», Sociologies pratiques, $\mathrm{n}^{\circ} 6$.

Berry, M. (1991), «Comment être jeune et consultant», Gérer et Comprendre, juin. 
Bourdieu, P. (1982), Leçon sur la leçon, Éditions de Minuit.

Brunsson, N. (2006), Mechanisms of Hope, Maintaining the Dream of the Rational Organization, Copenhagen Business School Press.

Davenport, H.T. (1995), «The fad that forgot people», L'Expansion Management Review.

Devan, J., Klusas, M.B., Ruefli, T.W. (2007), «The elusive goal of corporate outperformance», The McKinsey Quarterly, The Online Journal of McKinsey \& Co.

Goldratt, E., Cox, J. (1984), The Goal, North River Press.

Kimberly J.R. (dir.) (1980), "The poverty of imagery on organizational performance», dans The Organizational Life Cycle: Issues in the Creation, Transformation and Decline of Organizations, Jossey-Bass.

Midler, C. (1986), «Les modes managériales», Gérer et Comprendre, nº 4.

Mintzberg, H. (1989), Mintzberg on Management, Inside Our Strange World of Organizations, The Free Press.

Mintzberg, H. (2004), Managers not MBAs, Berrett-Koehler Publishers.

Morel, C. (2002), Sociologie des erreurs radicales et persistantes, Gallimard.

Moutout, J.-M. (2003), Violence des échanges en milieu tempéré, film, TS Production, France.

Nitin, N., Ranjay, G. (1997), «What is the optimum amount of organizational slack?», European Management Journal, vol. 15, $\mathrm{n}^{\circ}$ 6, décembre.

O'Shea, J., Madigan, C. (1997), Dangerous Company, Nicholas Brealey Publishing.

Perkins, J. (2004), Confessions of an Economic Hit Man, Penguin Books.

Power, M. (1997), The Audit Society: Rituals of Verification, Oxford University Press.

Schein, E.H. (1987), Process Consultation, vol. II. Lessons for Managers and Consultants, AddisonWesley.

Surowiecki, J. (dir.) (2002), Best Business Crime Writing of the Year, Random House.

Villette, M. (1988), L'homme qui croyait au management, Éditions du Seuil.

Villette, M. (2003), Sociologie du conseil en management, La Découverte. 